\title{
Sílica gel obtida de escória de alto forno: Marabá, Pará
}

\section{(Silica gel obtained from blast furnace slag: Marabá, PA, Brazil)}

\author{
M. M. Rebelo ${ }^{1^{*}}$, L. D. Nascimento ${ }^{2 *}$, J. A. M. Corrêa ${ }^{1}$ \\ ${ }^{1}$ Programa de Pós-Graduação em Geologia e Geoquímica - PPGG/IG, Instituto de Geociências \\ ${ }^{2}$ Programa de Pós-Graduação em Engenharia Química - PPEQ /ITEC, Instituto de Tecnologia \\ Universidade Federal do Pará, Av. Augusto Corrêa 01, Belém, PA Brasil 66075-110 \\ *Coordenação de Botânica, Museu Paraense Emílio Goeldi, Av. Perimetral 1901, Belém, PA, Brasil 66077-830 \\ monalizamaia@yahoo.com.br
}

\begin{abstract}
Resumo
Sílica gel com propriedades similares à sílica comercial foi obtida a partir de escória de alto forno (EAF), utilizando digestão com ácido clorídrico. A EAF-sílica obtida foi caracterizada por diferentes técnicas, mostrando-se amorfa, com pureza $99,7 \%$ e área específica $282 \mathrm{~m}^{2} / \mathrm{g}$. Apresentou caráter hidrofílico alto (12,27\%), com água de constituição de $\sim 6,18 \%$, o que foi confirmado pela perda de massa durante a análise termogravimétrica. As partículas de EAF-sílica apresentaram tamanhos micrométricos $(<1 \mu \mathrm{m})$ em forma de agregados, distribuição granulométrica unimodal e $\mathrm{D}_{50}$ $7,0 \mu \mathrm{m}$.
\end{abstract}

Palavras-chave: escória de alto forno, sílica gel, síntese.

\begin{abstract}
Silica gel with similar properties to commercial silica was obtained from blast furnace slag (EAF) using digestion with hydrochloric acid. The EAF-silica was obtained and characterized by different techniques. The results showed that the EAF-silica obtained was amorphous, with $99.7 \%$ purity and specific area $282 \mathrm{~m}^{2} / \mathrm{g}$. It presented high hydrophilic character $(12.27 \%)$, with constitution water of $\sim 6.18 \%$, which was confirmed by mass loss in thermogravimetric analysis. The EAF-silica particles had micrometric sizes $(<1 \mu \mathrm{m})$ with aggregates, unimodal size distribution and $D_{50}$ $7.0 \mu \mathrm{m}$.
\end{abstract}

Keywords: blast furnace slag, silica gel, syntheses.

\section{INTRODUÇÃO}

A indústria do aço no Brasil é uma das mais competitivas do mundo, sendo responsável por aproximadamente $18 \%$ do saldo comercial total do País, indicando o desenvolvimento econômico, social e ambiental do país, visto que o aço é matéria prima essencial em diferentes seguimentos [1]. Paralelamente, a crescente produção do aço reflete no aumento de geração de resíduos e coprodutos, inevitáveis ao processo industrial, pois de acordo com o Instituto Aço Brasil (IABR) [2], em 2011 foi produzido um total de 19,2 milhões de toneladas. Na produção de aço, as escórias representam cerca de $60 \%$ dos coprodutos, com o restante sendo composto por finos e pós (14\%), além de lamas, borras, refratários e outros, que juntos somam $26 \%$ do total.

A crescente geração de escórias apresenta sérios problemas, de tal modo que quantidades significativas delas estão dispostas no meio ambiente ou estocadas [2]; portanto, sua reciclagem pode ser uma alternativa para a fabricação de cimento, nivelamento de terrenos, base de estradas, concreto, argamassa, materiais agronômicos e de pavimentação [2-5]. A escória de alto forno (EAF), por exemplo, comporta-se de forma semelhante aos calcários, podendo ser utilizada como corretivo para a acidez do solo e ainda elevar as quantidades de $\mathrm{Ca}$ e $\mathrm{Mg}$, justificando sua utilização como fertilizante e como fonte de silício para as plantas $[6,7]$. A EAF também apresenta efeitos positivos no aumento da produtividade de arroz [6] e sua utilização permite a diminuição no consumo de inseticidas e fungicidas, pois, plantas bem nutridas com Si têm sua resistência às pragas e doenças aumentada [8]. Dessa forma, é importante buscar novas aplicações para esse coproduto, indo além do seu uso já comprovado como matéria prima para novos materiais - hidróxidos duplos lamelares $[9,10]$, visando um mercado promissor para ambos, além de poder garantir uma destinação final adequada para as escórias. As escórias são fontes abundantes de silicatos, sendo consideradas uma espécie de vidro de qualidade inferior à dos vidros comerciais; possuem elevado conteúdo em sílica, cujo teor total varia conforme o tipo de escória. A escória proveniente de aciaria tem uma percentagem em massa, de $\mathrm{SiO}_{2}$, que varia entre 8 e $22 \%$, contra uma variação de 27 a 
$43 \%$ para a escória de alto forno (EAF) $[9,11,12]$.

A sílica gel é um polímero inorgânico inerte, resistente, amorfo, com grande estabilidade térmica, mecânica e química, de extensa área específica e alta porosidade, de fácil moagem e altamente reativa [13]. Pode-se apresentar na forma pura [14], dopada [15] ou funcionalizada [16], sendo sintetizada por vários métodos, incluindo lixiviação química [17], processamento sol-gel [18] e sinterização no estado sólido [19]. O método mais empregado é o processo sol-gel, que permite o controle do crescimento das partículas coloidais de sílica, garantido características desejáveis como o diâmetro das esferas [14] e a área específica [20]. Dentre os tipos de sílica disponíveis no mercado, encontra-se o Aerosil ${ }^{\circledR}$, empregado em diferentes produtos comerciais usado em áreas de alta tecnologia, devido sua elevada pureza ( $>99 \%$ em massa de $\mathrm{SiO}_{2}$ ). Desse modo, a sílica gel se sobressai como um sólido poroso dotado de propriedades químicas e físicas específicas, podendo ser usada como matéria prima para fabricação de zeólitas [21], MCM [22], refratários [23], materiais de construção civil [24], pozolânicos [25], argamassa [26], entre outros. Quando sua superfície é quimicamente modificada por um agente organofuncionalizante [27], a sílica pode ser usada como suporte para catalisadores [28], fase estacionária em cromatografia líquida de alta eficiência (CLAE) [29] e para liberação controlada de fármacos [30], em tecnologias fotodinâmicas nas terapias do câncer [31] ou ainda como agente sequestrante de metais [32]. Visto que a EAF contém quantidades expressivas de sílica em sua composição, o presente trabalho realiza a caracterização da sílica proveniente da EAF, com o objetivo de determinar sua qualidade e propor possíveis destinos a esse material, avaliando parâmetros como tamanho de partícula, pureza química e área específica.

\section{MATERIAL E MÉTODOS}

Fonte de sílica: como fonte de sílica $\left(\mathrm{SiO}_{2}\right)$ utilizou-se a EAF fornecida de uma siderúrgica de Marabá, PA, Brasil. A escolha da EAF, classificada como ácida $\left(\mathrm{CaO} / \mathrm{SiO}_{2}<1\right)$, levou em consideração a sua abundância no meio ambiente e o elevado teor em $\mathrm{SiO}_{2}$ ( $>42 \%$ massa) em sua composição química. A quantidade de $\mathrm{SiO}_{2}$ fazia parte da estrutura cristalina da gehlenita e da pseudowollastonita, os dois minerais encontrados na EAF. Os percentuais mássicos dos demais elementos, na forma de óxidos, foram $\mathrm{CaO}$ : 41,42; $\mathrm{Al}_{2} \mathrm{O}_{3}: 13,32$; $\mathrm{MgO}: 1,07$ e $\mathrm{Fe}_{2} \mathrm{O}_{3}, \mathrm{TiO}_{2}, \mathrm{MnO}, \mathrm{K}_{2} \mathrm{O}, \mathrm{Na}_{2} \mathrm{O}$, $\mathrm{P}_{2} \mathrm{O} 5$ e $\mathrm{SO}_{3}$, com percentuais abaixo de $1 \%$, cada [9].

Obtenção da EAF-sílica: a EAF foi pulverizada em um moinho Retsch RMO, com granulação entre 70 e 250 mesh, e posteriormente digerida em ácido clorídrico de pureza analítica. Em um cadinho de teflon foram misturados a EAF com o ácido clorídrico a quente. Em seguida, à mistura foi adicionada água deionizada e o precipitado resultante foi filtrado a vácuo e lavado várias vezes com água deionizada, até neutralização. Posteriormente, o material obtido foi seco em temperatura ambiente e, então, codificado como EAFsílica. A parte solúvel deste processo de digestão ácida da EAF foi usada na síntese de hidróxidos duplos lamelares do tipo hidrotalcita [9].

Perda ao fogo da EAF-sílica: uma fração da amostra EAF-sílica foi dividida em duas partes. A primeira parte do material foi submetida a um aquecimento em estufa a 110 ${ }^{\circ} \mathrm{C}$ por $1 \mathrm{~h}$ para eliminação da água fisissorvida. O material restante não foi submetido ao pré-aquecimento. Em seguida, ambas as partes foram levadas, separadamente, a mufla em temperaturas entre $900 \mathrm{e} 1000^{\circ} \mathrm{C}$ por $1 \mathrm{~h}$ para determinação da água de constituição. A fração da EAF-sílica que foi submetida somente ao aquecimento em mufla foi codificada como EAF-sílica-M, enquanto o material pré-aquecido em estufa e posteriormente levado a mufla recebeu o código EAF-sílica-EM.

Caracterização dos materiais: a EAF-sílica foi caracterizada por fluorescência de raios $\mathrm{X}$, difração de raios $\mathrm{X}$, microscopia eletrônica de varredura, análises térmica diferencial e termogravimétrica, medida de área específica pelo método Brunauer, Emmett e Teller (BET) e distribuição de tamanho de partículas, método a laser. As amostras EAFsílica-EM e EAF-sílica-M foram caracterizadas somente por difração de raios X e determinação do tamanho de partículas.

Fluorescência de raios $X$ : a análise química da EAFsílica foi realizada em um espectrômetro sequencial AxiosMinerals, tubo de raios X cerâmico e anodo de Rh de 2,4 $\mathrm{kW}$ PANalytical. As concentrações dos elementos foram determinadas por meio do programa IQ+ Semiquant. A aquisição de dados foi feita com o software SuperQ Manager e o tratamento dos dados com o software IQ+, sendo o resultado normalizado para $100 \%$. A perda ao fogo foi calculada por diferença de massas após calcinação da amostra a $1000^{\circ} \mathrm{C}$.

Difração de raios $X$ : o equipamento utilizado foi um difratômetro PANalytical X'Pert Pro MPD, com goniômetro PW 3.050/60 $(\theta / \theta)$, tubo de raios $X$ cerâmico e anodo de cobre $\left(\mathrm{k} \alpha_{1}=1,540598 \AA\right)$. As análises foram realizadas em amostras na forma de pó, variando-se $2 \theta$ de 5 a $75^{\circ}$, voltagem $40 \mathrm{kV}$ e corrente $35 \mathrm{~mA}$, passo $0,02^{\circ} \mathrm{em} 2 \theta$ e $20 \mathrm{~s}$ o tempo/passo; fendas divergente de $1 / 8^{\circ}$ (para EAF-sílica) e automática (para EAF-sílica-EM e EAF-sílica-M) e antiespalhamento de $1 / 4^{\circ}$; máscara de $10 \mathrm{~mm}$. Os difratogramas foram interpretados digitalmente por comparação com arquivos ICCD.

Distribuição de tamanho de partícula: foi utilizado um analisador de partículas por difração a laser Analysette 22 MicroTec Plus, com amplitude de leitura de $0,08 \mu \mathrm{m}$ a 2000 $\mu \mathrm{m}$. As amostras foram preparadas em forma de suspensão, usando pirofosfato de sódio para auxiliar a dispersão do pó e, posteriormente, submetidas a agitação com ultrassom para a dispersão das partículas. Em seguida, alíquotas da 
suspensão do pó foram adicionadas e a homogeneização foi feita por um agitador mecânico do próprio equipamento. $\mathrm{O}$ comportamento da variação de distribuição granulométrica das amostras analisadas foi comparado pelo parâmetro $\mathrm{D}_{50}$, equivalente ao diâmetro teórico acima do qual há $50 \%$ de volume acumulado passante e $50 \%$ de volume acumulado retido da amostra considerada.

Microscopia eletrônica de varredura: as micrografias foram obtidas em um microscópio eletrônico de varredura LEO-1430, com as condições de análises para imagens de elétrons secundários: corrente do feixe de elétrons $90 \mu \mathrm{A}$, voltagem de aceleração constante $10 \mathrm{kV}$ e distância de trabalho $12-15 \mathrm{~mm}$. As amostras foram recobertas com um filme de platina.

Área especifica pelo método Brunauer, Emmett e Teller: as medidas de área específica da EAF-sílica foram feitas em um analisador Quantachrome Nova 1200, com adsorção e dessorção de nitrogênio a $77 \mathrm{~K}$ para cada superfície pelo método BET. Antes da adsorção de $\mathrm{N}_{2}$, as amostras foram submetidas a aquecimento por $2 \mathrm{~h}$ a $200^{\circ} \mathrm{C}$.

Análise térmica diferencial e termogravimétrica: a decomposição térmica e a estabilidade térmica da EAF-sílica foram avaliadas por análise termogravimetrica e análise térmica diferencial em equipamento Shimadzu DTG-60H, sob fluxo de nitrogênio entre 25 e $1000{ }^{\circ} \mathrm{C}$, com taxa de aquecimento $10{ }^{\circ} \mathrm{C} \cdot \mathrm{min}^{-1}$.

\section{RESULTADOS E DISCUSSÃO}

\section{Composição química da EAF-sílica}

$\mathrm{Na}$ Tabela I está apresentada a comparação entre a composição química da EAF-sílica e do Aerosil ${ }^{\circledR} 380$. A composição foi expressa em óxidos, segundo valores percentuais em massa. A EAF-sílica tem um elevado teor em $\mathrm{SiO}_{2}$, com valor superior a $93 \%$, seguido por $\mathrm{CaO}, \mathrm{Al}_{2} \mathrm{O}_{3}$, $\mathrm{K}_{2} \mathrm{O}, \mathrm{P}_{2} \mathrm{O}_{5}$ e $\mathrm{SO}_{3}$, que juntos representam apenas $0,24 \%$ do total da sua composição.

O conteúdo de 42,39 \% em $\mathrm{SiO}_{2}$ na EAF [9] já é considerado elevado, porém com a digestão da $\mathrm{EAF}$ em $\mathrm{HCl}$, foi possível obter sílica altamente pura. O procedimento de lavagem da EAF-sílica mostrou ser eficiente, restando apenas $0,09 \%$ de $\mathrm{HCl}$ residual na amostra. Na prática, o procedimento de digestão ácida da EAF, quando comparados com os processos de produção de sílicas comerciais, é bastante simples e muito satisfatório na obtenção de sílica pura, com ausência de subprodutos. As sílicas, por exemplo, do tipo Aerosil ${ }^{\circledR}$, são produzidas por um processo contínuo de hidrólise de clorosilanos $\left(\mathrm{SiCl}_{4}\right)$, em altas temperaturas. $\mathrm{O}$ Aerosi $1^{\circledR}$ é um tipo de sílica pirogênica, considerada uma das mais puras disponíveis no mercado. O processo de obtenção da sílica pirolizada envolve muitas etapas, dentre as quais há formação de ácido clorídrico gasoso como subproduto. Este processo é bastante complexo quando comparado ao
Tabela I - Comparação entre a composição química da EAFsílica e do Aerosil ${ }^{\circledR} 380$.

[Table I - Comparison between the chemical composition of BFS-silica and Aerosil ${ }^{\circledR}$ 380.]

\begin{tabular}{cccc}
\hline Constituintes & $\begin{array}{c}\text { EAF-sílica } \\
\text { \%-massa }\end{array}$ & $\begin{array}{c}\text { EAF-sílica } \\
\text { (calc.) } \\
\text { \%-massa }\end{array}$ & $\begin{array}{c}\text { Aerosi }{ }^{\circledR} 380 \\
\text { (calc.) } \\
\text { \%-massa }\end{array}$ \\
\hline $\mathrm{SiO}_{2}$ & 93,49 & 99,65 & 99,80 \\
$\mathrm{CaO}$ & 0,10 & 0,11 & n.d. \\
$\mathrm{Al}_{2} \mathrm{O}_{3}$ & 0,06 & 0,06 & 0,05 \\
$\mathrm{~K}_{2} \mathrm{O}$ & 0,05 & 0,05 & $n . d$. \\
$\mathrm{P}_{2} \mathrm{O}_{5}$ & 0,01 & 0,01 & n.d. \\
$\mathrm{SO}_{3}$ & 0,02 & 0,02 & $n . d$. \\
$\mathrm{HCl}_{2}$ & 0,09 & 0,10 & 0,03 \\
$\mathrm{Fe}_{2} \mathrm{O}_{3}$ & n.d. & n.d. & 0,003 \\
$\mathrm{TiO}_{2}$ & n.d. & n.d. & 0,03 \\
$\mathrm{PF}^{*}$ & 6,18 & n.d. & n.d. \\
\hline $\mathrm{Total}$ & 100 & 100 & 99,91 \\
\hline
\end{tabular}

PF: Perda ao fogo; n.d.: não detectado; calc.: amostra calcinada, em base livre de umidade.

PF: Loss on Ignition; n.d.: not detected; calc.: calcined sample, moisture free basis.

descrito no presente trabalho. Ainda referente à Tabela I, observa-se um elevado teor de perda ao fogo, $6,18 \% \mathrm{em}$ massa, que pode ser um fator atenuante da quantidade da sílica obtida, pois está diretamente relacionado ao teor de água de constituição da EAF-sílica, já que não foram detectados teores significativos de carbono na amostra. Este valor de água de constituição da EAF-sílica encontra-se bem acima dos valores encontrados nas sílicas Aerosil ${ }^{\circledR}$. A pureza da EAF-sílica é compatível àquelas encontradas nas sílicas comerciais. Considerando a amostra calcinada (base livre de umidade), a EAF-sílica apresenta um conteúdo mássico de $\mathrm{SiO}_{2}$ igual a $99,7 \%$, muito semelhante àquele encontrado no Aerosil ${ }^{\circledR} 380$ (99,8\%). Os demais elementos, encontrados na EAF-sílica, mostram concentrações relativamente baixas.

\section{Composição mineralógica da EAF-sílica}

Uma característica bastante importante relacionada à reatividade das sílicas diz respeito a sua amorficidade. No difratograma de raios $\mathrm{X}$ da EAF-sílica observa-se que a curva, com suave inclinação e sem presença de picos, evidencia a ausência de cristais, ou seja, a EAF-sílica apresenta-se amorfa (Fig. 1). O acentuado background para todas as posições entre $15<2 \theta<32^{\circ} 2 \theta$ é típico de sílica amorfa, em que o dióxido de silício é predominante, estando os demais óxidos presentes, provavelmente, sob a forma de solução sólida [33].

As amostras de EAF-sílica calcinadas a $1000^{\circ} \mathrm{C}$, também foram caracterizadas por difração de raios $X$ (Fig. 2). Observou-se que a amostra EAF-sílica-M, não submetida ao aquecimento prévio, se manteve amorfa. Porém, na amostra EAF-sílica-EM, observa-se um pequeno pico em 26,7, 


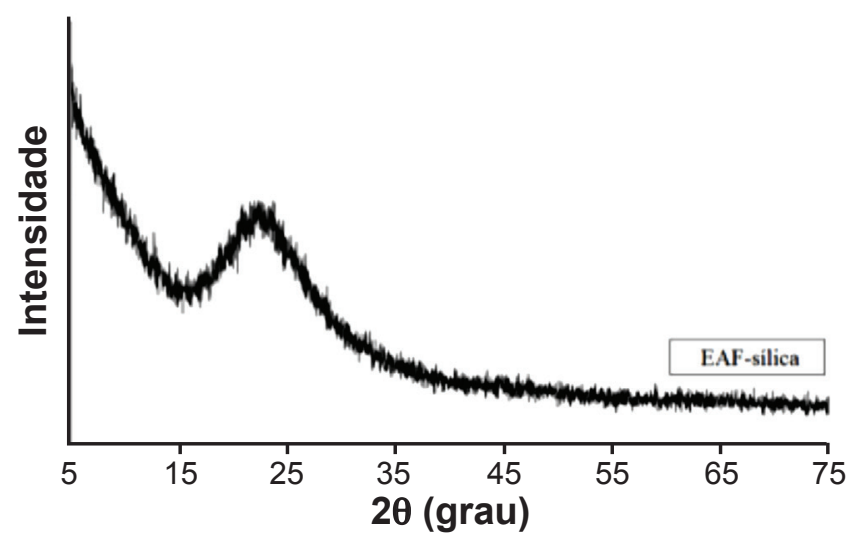

Figura 1: Difratograma de raios $\mathrm{X}$ da amostra EAF-sílica, sem tratamento. Notar o padrão típico de material com elevada amorficidade.

[Figure 1: X-ray diffraction pattern of untreated BFS-silica. Note the typical pattern of material with high amorphicity.]

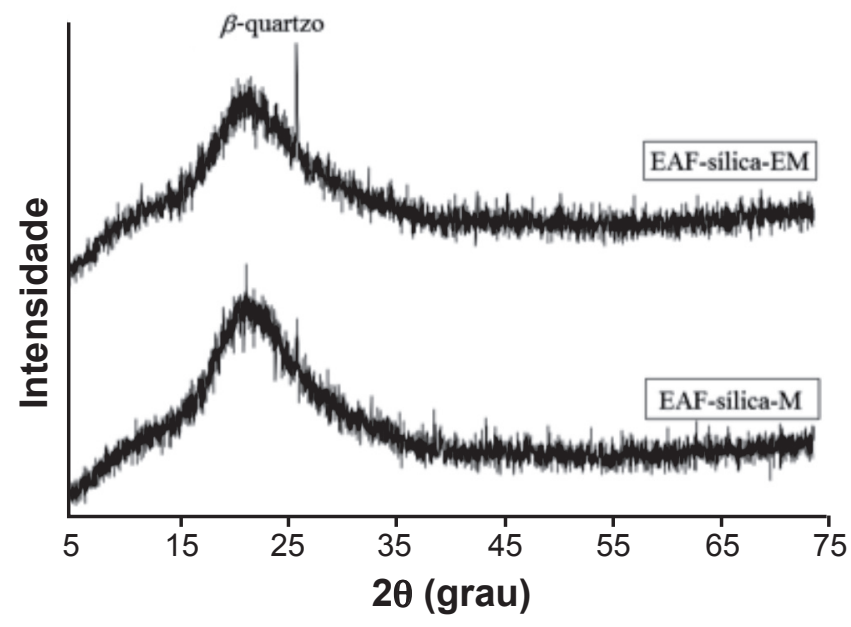

Figura 2: Difratogramas de raios $\mathrm{X}$ da EAF-sílica-EM e EAFsílica-M após calcinação a $1000{ }^{\circ} \mathrm{C}$.

[Figure 2: X-ray diffraction patterns of BFS-silica-EM and BFSsilica-M, after calcination at $1000^{\circ} \mathrm{C}$.]

relacionado à reflexão (011), associado à estrutura hexagonal do $\beta$-quartzo (arquivo PDF 01-085-0795). O aparecimento deste pico pode estar associado ao aquecimento da amostra a $110{ }^{\circ} \mathrm{C}$ por $1 \mathrm{~h}$, pois este tipo de tratamento térmico permite a saída e evaporação dos voláteis, proporcionando a estruturação da rede vítrea devido à presença de óxidos formadores de rede, neste caso o $\mathrm{SiO}_{2}$ [34].

Existem estudos em que a forma $\beta$-quartzo aceita combinações de íons menores, tais como: $\mathrm{Li}^{+}, \mathrm{Be}^{2+}, \mathrm{Mg}^{2+}$, $\mathrm{Zn}^{2+}, \mathrm{Al}^{3+}$ formando, portanto, variadas composições. Adições de certos tipos de óxidos, como por exemplo o de $\mathrm{Ca}, \mathrm{Mg}$ e Al, estabilizam a fase formada, evitando transformações de fase durante o aquecimento e o resfriamento [34, 35]. A ausência de fases mais estáveis a altas temperaturas, quando a EAF-sílica foi aquecida até $1000^{\circ} \mathrm{C}$, pode estar associada à presença de pequenas quantidades de impurezas $(<0,25 \%$ massa). Portanto, se faz necessário um aquecimento na EAFsílica acima de $1000{ }^{\circ} \mathrm{C}$ para obtenção de fases mais estáveis como cristobalita e tridimita. A formação de cristobalita a partir de uma sílica amorfa sempre apresenta uma transformação de fase consideravelmente mais lenta que aquela obtida por meio da inversão do quartzo [33]. Os resultados obtidos de difração de raios $\mathrm{X}$ das amostras analisadas estão de acordo com os reportados [33]. Foram avaliadas as transformações de fases em sílicas contendo impurezas e observado que o $\boldsymbol{\alpha}$-quartzo é a forma mais estável de $\mathrm{SiO}_{2}$ na temperatura ambiente e que a $573{ }^{\circ} \mathrm{C}$ transforma-se em $\beta$-quartzo. Foi verificado também que a presença de fases como $\beta$-cristobalita ou $\beta$-tridimita, somente foi possível quando o aquecimento foi acima de $1000{ }^{\circ} \mathrm{C}$. Acima de $1470{ }^{\circ} \mathrm{C}$ foi observada a reversibilidade $\beta$-cristobalita $\leftrightarrow \beta$-tridimita e acima de $1710^{\circ} \mathrm{C}$ tem-se $\beta$-tridimita $\leftrightarrow$ sílica glass. Foaram também estudadas as transformações de fases de sílica e observado que além da presença de pequenas quantidades de impurezas, estas transformações eram tanto influenciadas pela taxa de aquecimento e resfriamento, quanto pelos efeitos de tamanho de partícula e condições de superfície [36].

Distribuição de tamanho de partículas da EAF-sílica antes e após a calcinação

A Fig. 3 mostra as curvas de distribuição granulométrica da (a) EAF-sílica e (b) EAF-sílica-EM. A percentagem em volume é expressa para distribuição cumulativa $\mathrm{Q} 3(\mathrm{X})$ e diferencial dQ3(X), assumindo que as partículas
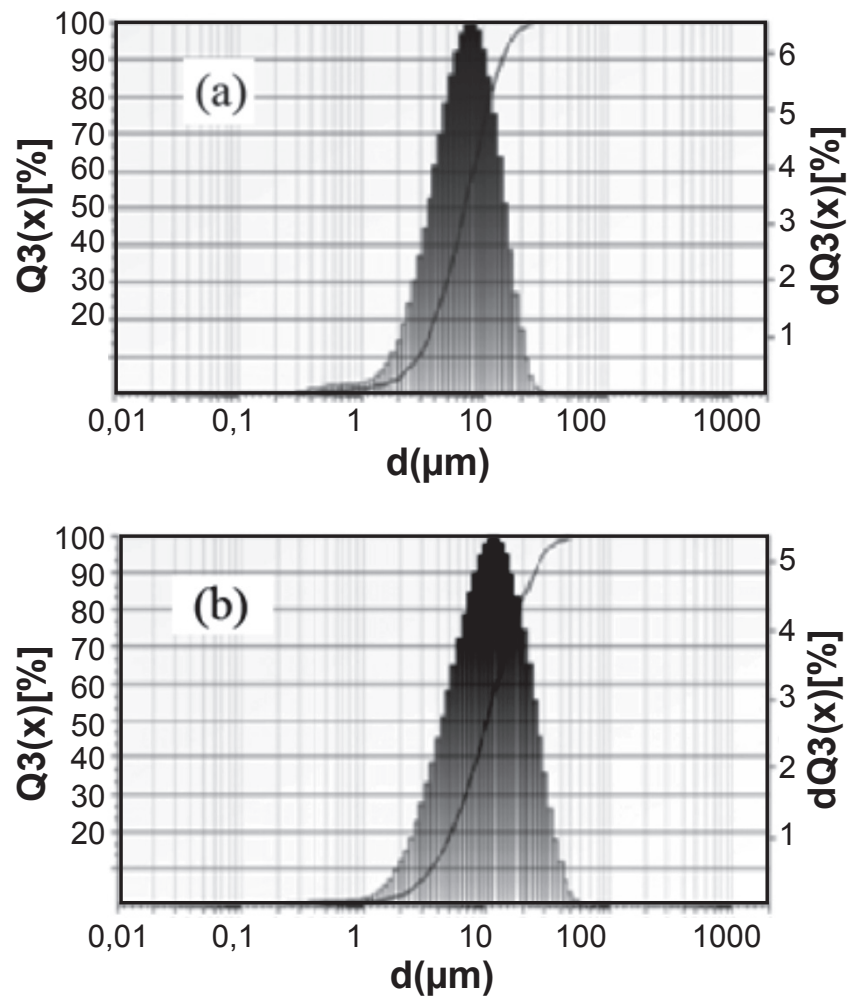

Nota-Q3(X): Distribuição cumulativa e dQ3(X) Distribuição de frequência. $Q 3(X)$ Cumulative distribution and $d Q 3(X)$ : Frequency distribution.

Figura 3: Curvas de distribuição granulométrica de (a) EAF-sílica e (b) EAF-sílica-EM, obtida por difração a laser.

[Figure 3: Particle size distribution of (a) BFS-silica and (b) BFSsilica-EM, obtained by laser diffraction.] 
são esféricas. As curvas têm comportamento gaussiano e distribuição unimodal, com tamanho de partículas correspondente ao diâmetro teórico $\mathrm{D}_{50}$ de $7,0 \mu \mathrm{m}$ e $10,2 \mu \mathrm{m}$, atribuídos a EAF-sílica e EAF-sílica-EM, respectivamente. Estes dados encontram-se na Tabela II, juntamente com os diâmetros $\mathrm{D}_{10}$ e $\mathrm{D}_{90}$ e seus diâmetros médios.

Observa-se ainda, na Fig. 3 que as amostras apresentamse homogêneas, com diâmetros de partículas variando

Tabela II - Valores de $\mathrm{D}_{10}, \mathrm{D}_{50}$ e D90 e diâmetros médios das amostras EAF-sílica e EAF-sílica-EM.

[Table II - Values of $D_{10}, D_{50}$ and $D_{90}$ of BFS-silica and BFSsilica-EM and their average diameters.]

\begin{tabular}{ccc}
\hline Diâmetro teórico $(\mu \mathrm{m})$ & EAF-sílica & EAF-sílica-EM \\
\hline $\mathrm{D}_{10}$ & 2,9 & 3,6 \\
$\mathrm{D}_{50}$ & 7,0 & 10,2 \\
$\mathrm{D}_{90}$ & 14,0 & 23,8 \\
$\mathrm{DM}$ & 8,0 & 12,5 \\
\hline
\end{tabular}

DM: Diâmetro médio. DM: mean diameter
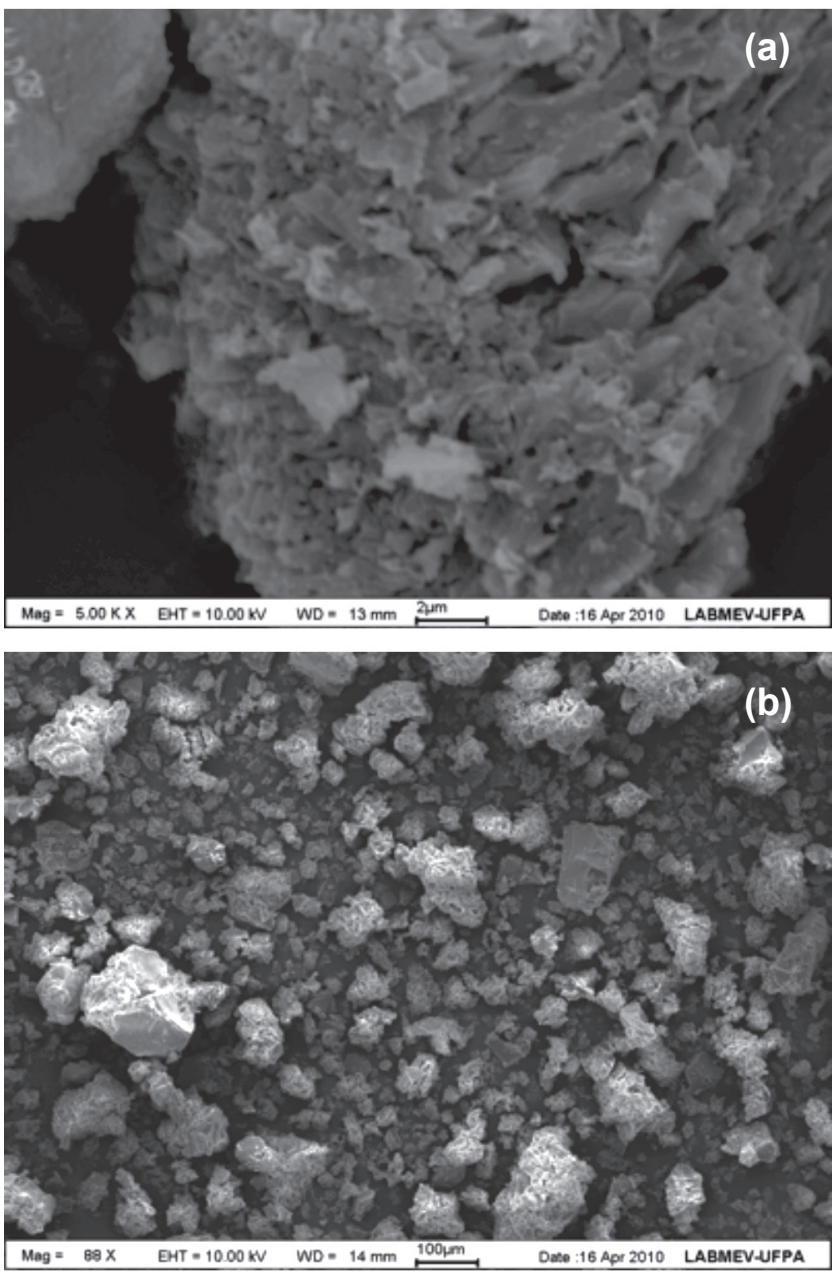

Figura 4: Micrografias obtidas em microscópio eletrônico de varredura de amostras de EAF-sílica, imagens obtidas por elétrons secundários com magnitudes de (a) $5.000 \times$ e (b) $88 \mathrm{x}$.

[Figure 4: SEM micrographs of BFS-silica, obtained by secondary electron with magnitudes of (a) 5000x and (b) 88x.] dentro de uma faixa bastante estreita. Para a EAF-sílica, o diâmetro de partículas varia de 0,7 a $30,0 \mu \mathrm{m}$, enquanto que o diâmetro da EAF-sílica-EM varia de $0,7 \mu \mathrm{m}$ a $66,0 \mu \mathrm{m}$. Analisando estes dados, sugere-se que a amostra EAF-sílica, sem tratamento térmico, apresenta-se mais homogênea, com seu diâmetro de partículas variando em uma faixa ainda mais estreita. Os dados contidos na Tabela II mostram que a EAF-sílica-EM apresenta sempre os maiores diâmetros de partículas $\left(\mathrm{D}_{10}: 3,6 ; \mathrm{D}_{50}: 10,2\right.$ e $\left.\mathrm{D}_{90}: 23,8 \mu \mathrm{m}\right)$ quando comparados a EAF-sílica $\left(\mathrm{D}_{10}: 2,9 ; \mathrm{D}_{50}: 7,0\right.$ e $\left.\mathrm{D}_{90}: 14,0 \mu \mathrm{m}\right)$. Estes resultados sugerem que a amostra quando submetida à calcinação, tem seus diâmetros de partículas aumentados. A EAF-sílica em água não é totalmente dispersa. $\mathrm{O}$ uso de pirofosfato de sódio e ultrassom promoveu acentuada redução no diâmetro das partículas em forma de aglomerado $\left(\mathrm{D}_{50} \sim 7,0\right.$ a $\left.10,2 \mu \mathrm{m}\right)$. Contudo, a desaglomeração das partículas não foi completa, prevalecendo aglomerados com diâmetros médios muito maiores quando comparados ao diâmetro médio de partículas primárias de sílica Aerosil ${ }^{\circledR}$ $(7,0 \mathrm{~nm}$ a $40,0 \mathrm{~nm})$. O diâmetro médio em volume do Aerosil $^{\circledR} 200$ é $\mathrm{D}_{50}: 22,0 \mu \mathrm{m}[37]$.

\section{Aspectos morfológicos da EAF-sílica}

Nas micrografias obtidas por MEV é observada a formação de agregados microscópicos característicos de material sinterizado $(<1 \mu \mathrm{m})$, visualizados na Fig. 4a. $\mathrm{Na}$ Fig. $4 \mathrm{~b}$ nota-se que esses agregados são de tamanhos variados, tanto menores como maiores que $100 \mu \mathrm{m}$. Essa avaliação reforça que é necessário um equipamento com resolução ainda mais alta para conseguir visualizar o tamanho de partícula primária da EAF-sílica.

Nas Figs. 5 e 6 é possível visualizar que a EAF-sílica apresenta-se tanto na forma não densificada, com grânulos de 10 a $20 \mu \mathrm{m}$, quanto densificada, apresentando grânulos

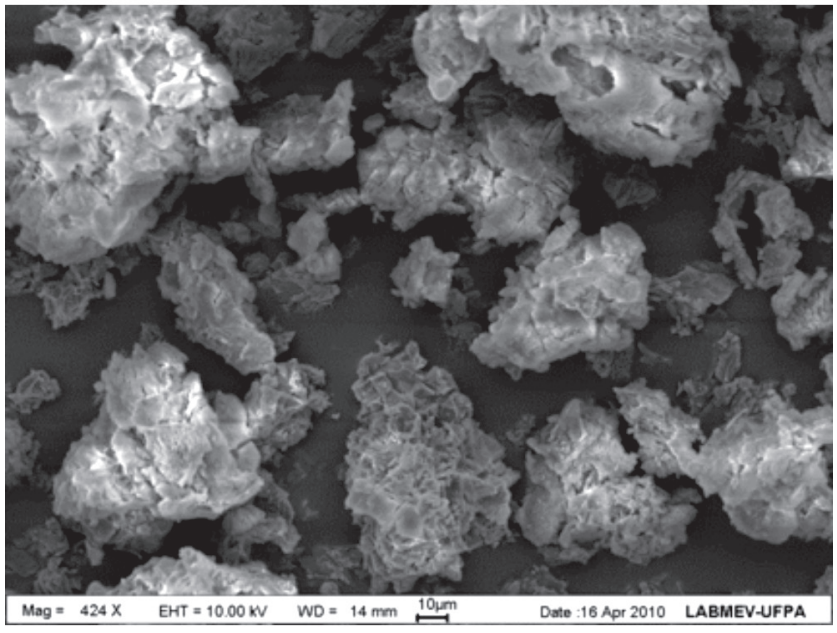

Figura 5: Micrografia obtida em microscópio eletrônico de varredura de EAF-sílica, na forma não densificada. Notar aglomerados com tamanhos entre 10 e $20 \mu \mathrm{m}$.

[Figure 5: SEM micrograph of non-densified BFS-silica. Agglomerates in the range 10-x20 $\mu \mathrm{m}$.] 

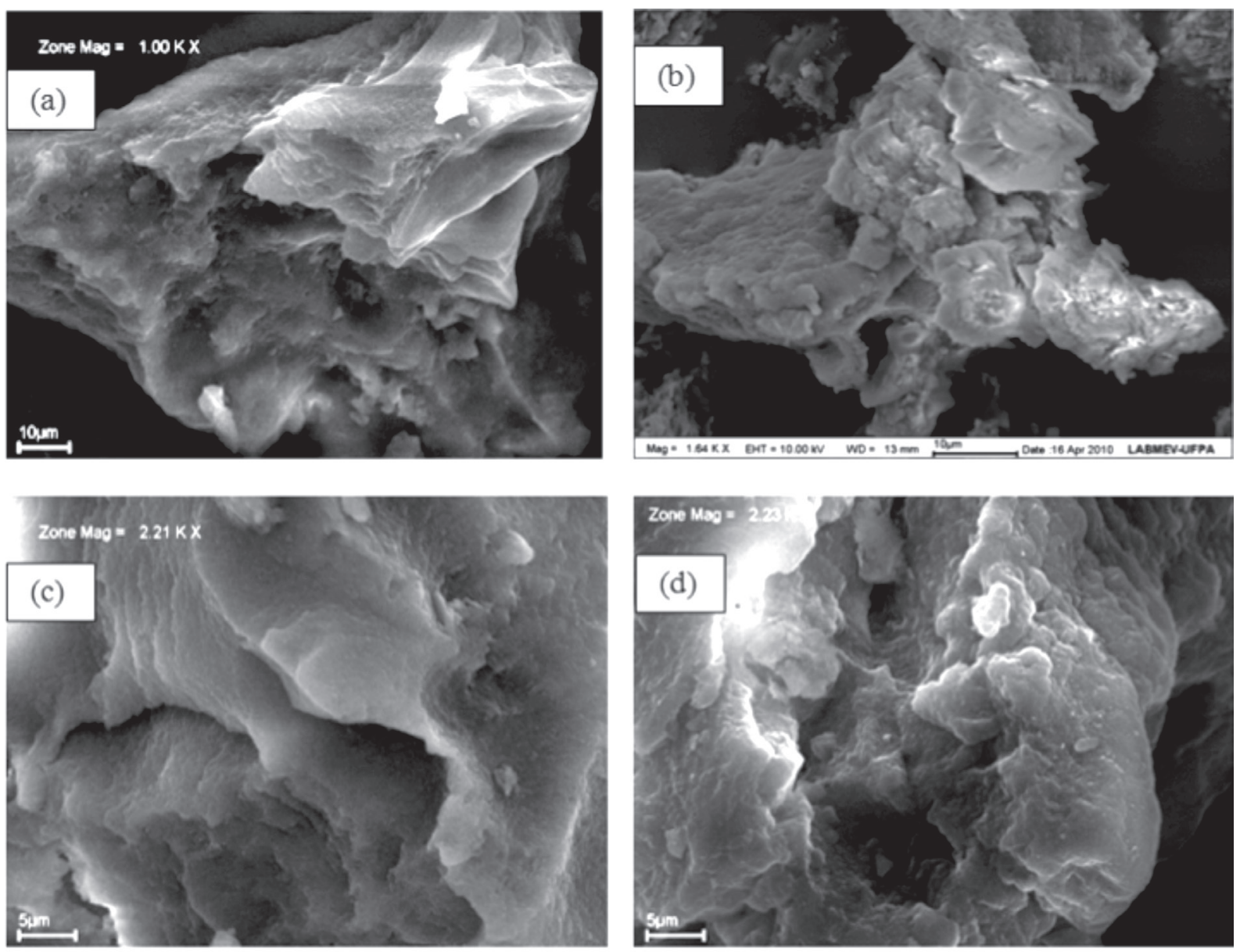

Figura 7: Micrografias obtidas em microscópio eletrônico de varredura de EAF-sílica, imagens obtida por elétrons secundários com magnitudes de (a) 1000×, (b) 1640x, (c) 2210x e (d) 2230x.

[Figure 7: SEM micrographs of BFS-silica; secondary electrons. (a) 1.000x, (b) 1640x, (c) 2210x and (d) 22300x.]

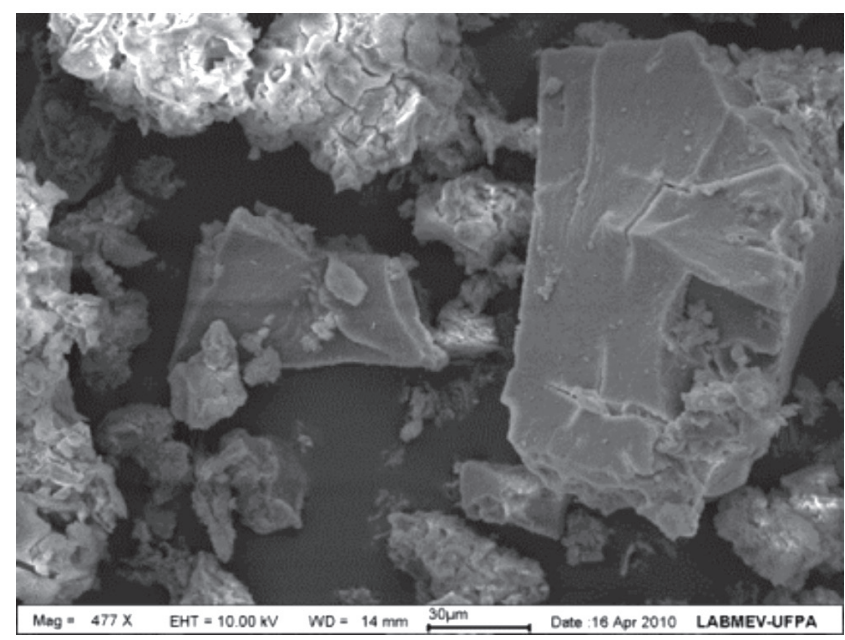

Figura 6: Micrografia obtida em microscópio eletrônico de varredura EAF-sílica, na forma densificada. Notar aglomerados com tamanhos maior que $100 \mu \mathrm{m}$.

[Figure 6: SEM micrograph of non-densified BFS-silica. Agglomerates larger than $100 \mu \mathrm{m}$.

$>100 \mu \mathrm{m}$. A sílica não densificada é responsável pela sua maior atividade pozolânica, enquanto a forma densificada, é benéfica quanto ao manuseio, transporte e armazenamento do material [38].

A forma como os agregados estão arranjados permite visualizar (Figs. 7a a 7d) a EAF-sílica como um material com aspecto superficial semelhante àqueles com estrutura quebradiças e porosas, tal como as sílicas Aerosil ${ }^{\circledR}$, com considerável área específica.

\section{Área de superfície especifica}

A área específica (BET) da EAF-sílica foi $282 \mathrm{~m}^{2} / \mathrm{g}$, compatível com aquelas encontradas nas sílicas comerciais. Dentre os diversos tipos de Aerosil ${ }^{\circledR}$, cujas áreas específicas variam de $50 \mathrm{~m}^{2} / \mathrm{g}$ a $380 \mathrm{~m}^{2} / \mathrm{g}$, o Aerosil ${ }^{\circledR} 200$ tem $200 \mathrm{~m}^{2} / \mathrm{g}$ e é conhecido como um dos melhores produtos. A EAFsílica, com maior área específica que o Aerosil ${ }^{\circledR} 200$, pode ser utilizada para as mesmas finalidades que este produto já comercializado. Em virtude da sua alta área específica, a EAF-sílica pode adsorver substâncias voláteis do ambiente. Além disso, a superfície da EAF-sílica pode ser modificada, por vários grupos ligados quimicamente, deixando-a com características hidrofílicas ou hidrofóbicas, aliadas as mais diversas aplicações. 


\section{Termogravimetria da EAF-sílica}

A termodecomposição da EAF-sílica está mostrada na Fig. 8. A curva termogravimétrica apresenta três etapas de perda de massa. A perda de massa total foi $18,93 \%$.

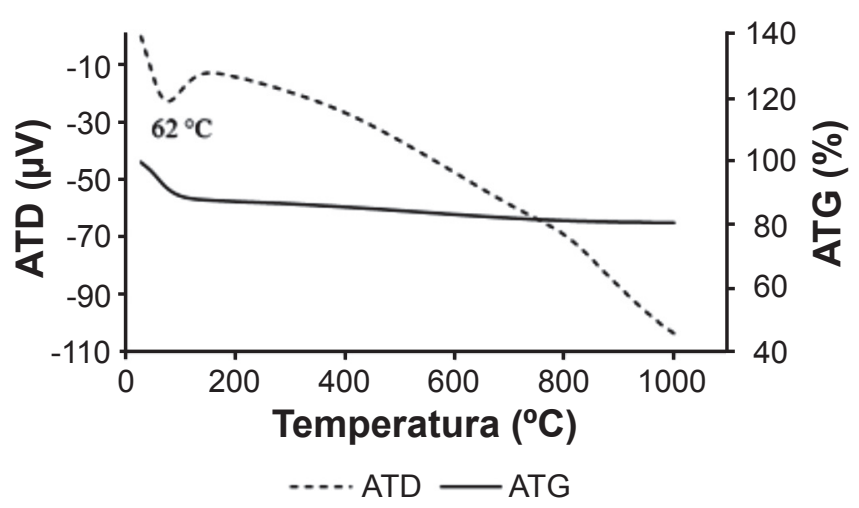

Figura 8: Curva das análises térmica diferencial e termogravimétrica da EAF-sílica, sem tratamento.

[Figure 8: Curve of differential thermal analysis and thermogravimetric BFS-silica untreated.]

A maior taxa de perda de massa $12,27 \%$, evidenciada na curva TG ocorre no intervalo entre 28 e $220{ }^{\circ} \mathrm{C}$, coincidindo com a temperatura do único pico endotérmico da curva de análise térmica diferencial, com valor máximo na temperatura próxima de $62{ }^{\circ} \mathrm{C}$. A esta primeira etapa de perda de massa foi atribuída à saída da água fisisorvida na superfície da EAFsílica. Na segunda etapa, observa-se entre 220 e $600^{\circ} \mathrm{C}$, que o material apresenta uma perda de massa de 3,97\%, devido à desidroxilação reversível dos grupos silanóis. $\mathrm{Na}$ terceira etapa, que ocorre entre 600 a $100{ }^{\circ} \mathrm{C}$, há uma perda de massa de $2,66 \%$, relacionada à desidroxilação total e irreversível na superfície do óxido. Na curva TG, a perda de massa $6,63 \%$, no intervalo de temperatura de 220 até $1000{ }^{\circ} \mathrm{C}$ é semelhante ao resultado do experimento de perda ao fogo $(6,18 \%)$. Com estes dados, sugere-se que devido a EAF-sílica apresentar elevada pureza, com alto teor em $\mathrm{SiO}_{2}$, tenha-se elevada quantidade de água de constituição. As amostras de sílica amorfa com maior proporção em Si têm mais água ocluída, devido a condensação das ligações -Si-OH----HO-Si-OH [39]. No termograma apresentado não foi observado pico exotérmico, característico de formação de fase cristalina, como o quartzo, observado no difratograma da amostra EAF-sílica-EM. Este fato pode estar relacionado à atmosfera de $\mathrm{N}_{2}$, a qual a amostra foi submetida para realização desta análise térmica. A desidroxilação da superfície da EAF-sílica é parecida àquela observada [40], observando que a desidroxilação da superfície da sílica acontecia em mais de uma etapa. Aquecendo-a entre 100 e $150{ }^{\circ} \mathrm{C}$ removia-se basicamente a água fisisorvida e acima desta temperatura iniciava-se a condensação dos grupos silanóis, produzindo água. Devido à capacidade de fisisorver consideráveis quantidades de água (12,27\%), a EAF-sílica mostra-se como um material promissor para ser usado como agente desumidificante, além de aplicações em áreas de alta tecnologia.

\section{CONCLUSÕES}

Ficou constatado, como primeiro relato, que no Brasil (Pará), conseguiu-se obter sílica gel por meio da digestão ácida da escória de alto forno, proveniente da cidade de Marabá. O processo aqui descrito, quando comparado com aqueles utilizados no preparo das sílicas comerciais, é relativamente mais simples, econômico e não envolve formação de subprodutos. A EAF-sílica obtida apresenta um diâmetro teórico $\mathrm{D}_{50}$ de $7 \mu \mathrm{m}$ e outras características semelhantes às encontradas nas sílicas comerciais, tais como elevadas amorficidade, estabilidade térmica, área específica, pureza química, bem como alto caráter hidrofílico e conteúdo de água ocluída em sua composição química, tornando-a promissora nas mais importantes áreas de ciência, tecnologia e inovação. Uma vez modificada a superfície da EAF-sílica, por grupos orgânicos ou funcionais ligados quimicamente, esta pode apresentar um potente desempenho como fase estacionária de técnicas cromatográficas, além de poder ser usada como suporte para catalisadores, agente sequestrante de metais e para liberação controlada de fármacos.

\section{AGRADECIMENTOS}

Ao CNPq pela bolsa de doutorado, ao Programa de Pós Graduação em Geologia e Geoquímica da UFPA pelo uso dos laboratórios de Análises Químicas, XRPD, FRX, MEV, DTP e ao Programa de Pós Graduação em Química da UFPA pelo uso dos laboratórios de DTA, TG e BET. Os autores agradecem, em especial, ao químico Natalino Valente pelo apoio no laboratório de análises químicas do PPGG-UFPA.

\section{REFERÊNCIAS}

[1] Instituto Aço Brasil, acesso em 12/10/12, disponível em http://www.acobrasil.org.br/site/portugues/ instituto/ marca. asp>

[2] Instituto Aço Brasil, acesso em 15/10/12, disponível em $<$ http://www.acobrasil.org.br/site/ portugues/ biblioteca/ relatorio_sustentabilidade_2012.pdf $>$

[3] V. A. S Ribeiro, A. L. da Silva, M. R. da Silva, J. C. Dias, Rev. Matéria 12, 2 (2007) 339.

[4] A. S. Liduário, L. dos A. Farias, A. C. Albuquerque, M. A. S. Andrade, Tecnol. Metal. Mater. 2, 1 (2005) 35.

[5] Associação Brasileira de Metalurgia e Materiais, Gestão de Coprodutos: Estudo Prospectivo do Setor Siderúrgico (Nota Técnica) (2008) 14.

[6] J. G. C. Pupatto, L. T. Büll, C. A. C. Crusciol, M. Mauad, R. H. Silva, Pesq. Agropec. Bras. 38 (2003) 1323.

[7] J. G. C. Pupatto, L. T. Büll, C. A. C. Crusciol, Pesq. Agropec. Bras. 39 (2004) 1213.

[8] G. H. Korndörfer, L. E. Datnoff, "Adubação com silício: uma alternativa no controle de doenças da cana-de açúcar e do arroz", Inf. Agron., Piracicaba, SP (1995).

[9] M. M Rebelo, M. V. P. O Cunha, J. A. M. Corrêa, Quim. Nova 35, 5 (2012) 883.

[10] Y. Kuwahara, T. Ohmichi, T. Kamegawa, K. Mori, H. 
Yamashita, J. Mater. Chem. 20 (2010) 5052.

[11] E. A. Polisseni, Tese Dr., Universidade Federal do Rio Grande do Sul, Brasil (2005).

[12] W. Mendes, Diss. Mestrado, Universidade Estadual de Campinas, Brasil (1999).

[13] N. N. Greenwood, A. Earnshaw, "Chemistry of the Elements", $2^{\text {nd }}$ ed., Oxford, Butterworth-Heinemann (2002). 328

[14] H. Izutsu, F. Mizukami, P. K. Nair, Y. Kiyozumi, K. Maeda, J. Mater. Chem. 7, 5 (1997) 767.

[15] D. C. M. Dutoit, M. Schneider, P. Fabrizioli, A. Baiker, J. Mater. Chem. 7, 2 (1997) 271.

[16] A. R. Cestari, C. Airoldi, Langmuir 13 (1997) 2681.

[17] N. Yalçin, V. Sevinç, Ceram. Int. 27 (2001) 219.

[18] R. F. S. Lenza, W. L. Vasconcelos, Quim. Nova 25, 6 (2002) 893.

[19] O. Yong-Taeg, S. Fujino, K. Morinaga, Sci. Technol. Adv. Mater. 3 (2002) 297.

[20] G. Zehl, S. Bischoff, F. Mizukami, H. Isutzu, M.

Bartoszek, H. Jancke, B. Lucke, K. Maeda, J. Mater. Chem. 5 (1995) 1893.

[21] A. Caovilla, R. T. Rigo, F. G. Penha, S. B. C. Pergher, Quim. Nova 32 (2009) 1818.

[22] H. I. Meléndez-Ortiz, A. Mercado-Silva, L. A. GarcíaCerda, G. Castruita, Y. A. Perera-Mercado, J. Mex. Chem. Soc. 57, 2 (2013) 73.

[23] V. P. Della, I. Kühn, D. Hotza, Quim. Nova 24 (2001) 778.

[24] J. B. M. Carmo, K. F. Portella, Cerâmica 54 (2008) 309.

[25] G. C. Cordeiro, R. D. T. Filho, E. de M. R. Fairbairn, Quim. Nova 32 (2009) 82.

[26] I. M. T. Bezerra, J. Souza, J. B. Q Carvalho, G. A.
Neves, Rev. Bras. Eng. Agr. Amb. 15 (2011) 639.

[27] L. N. H. Arakaki, C. Airoldi, Quim. Nova 22 (1999) 246.

[28] V. Polshettiwar, C. Len, A. Fihri, Coordination Chem. Rev. 253 (2009) 2599.

[29] C. R. Silva, I. C. S. F. Jardim, C. H. Collins, C. Airoldi, Quim. Nova 27 (2004) 270.

[30] W. R. Braz, A. L. A. Moura, E. F. Molina, N. L. Rocha, E. H. de Faria, O. S. Calefi, K. J. Ciuffi, L. A. Rocha, E. J. Nassar, Rev. Ci. Farmac. Bas. Apl. 35 (2014).

[31] T. Y. Ohulchanskyy, I. Roy, L.N. Goswami, Y. Chen, E.J. Bergey, R.K. Pandey, A.R. Oseroff, P.N. Prasad, Nano Lett. 7 (2007) 2835.

[32] C. Airoldi, R. F. Farias, Quim. Nova 23 (2000) 496.

[33] F. M. Wahl, R. E. Grim, R. B. Graf, The Am. Mineralogist 46 (1961) 196.

[34] R. H. Doremus, "Glass Science", $2^{\text {nd }}$ ed., John Wiley \& Sons, Nova York, EUA (1994) 352p.

[35] J. M. F. Navarro, "El Vidrio - Manual sobre ciencia y tecnología del vidrio, de caracter didáctico y enfoque científico", Fundación Centro Nacional del Vidrio, Madri, Espanha (1991) 696p.

[36] F. A. Hummel, "Introduction to Phase Equilibria in Ceramics Systems", Marcel Dekker, Inc., EUA (1984) 30.

[37] G. S. Domingues, S. S. Guterres, R. C. R. Beck, A. R. Pohlmann, Quim. Nova 31 (2008) 1966.

[38] M. I. S. Rojas, J. Rivera, M. M. Frias, Cement Concrete Res. 29 (1999) 945.

[39] F. Touati, F. Sediri, N. Gharbi, Mater. Chem. Phys. 101 (2007) 352.

[40] R. K. Iler, "The Chemistry of Sílica", John Wiley \& Sons, New York, EUA (1979) 896p.

(Rec. 24/12/2014, Rev. 01/03/2015, Ac. 30/04/2015) 